CLINICAL STUDY

\title{
Prevalence of positive anti-neutrophil cytoplasmic antibody (ANCA) in patients receiving anti-thyroid medication
}

\author{
Jenny E Gunton, John Stiel, Phillip Clifton-Bligh, Errol Wilmshurst and Aidan McElduff \\ Department of Endocrinology, Royal North Shore Hospital, St Leonards, Sydney, Australia \\ (Correspondence should be addressed to J E Gunton; Email: jennyg@easy.com.au)
}

\begin{abstract}
Objective: Vasculitis is a rare complication of anti-thyroid medications. There are 32 cases of antineutrophil cytoplasmic antibody (ANCA)-positive vasculitis in association with anti-thyroid medication reported in the English literature. The objectives of this study were to assess the frequency of positive ANCA in patients on long-term anti-thyroid medication, and to follow patients prospectively from commencement of medication to determine whether they became ANCA-positive after therapy. Design: Prospectively collected cross-sectional study of two groups of patients: (i) who had received long-term (>18 months) anti-thyroid medication, and (ii) newly diagnosed thyrotoxicosis before commencement of anti-thyroid medication attending clinic between 28 April 1998 and 30 September 1998. Data were collected for age, sex, ethnicity, underlying thyroid disease, medication and duration, and symptomatology.

Results: Eight of 30 patients on long-term anti-thyroid medication $(26.7 \%)$ were ANCA-positive. All ANCA-positive patients were female, seven were taking propylthiouracil (PTU) at the time of testing. ANCA-positive patients had taken PTU for a mean \pm s.D. of $7.9 \pm 10.2$ years, compared with $0.8 \pm 2.2$ years in ANCA-negative patients (Mann-Whitney, $P<0.0001$ ). The ten patients with newly diagnosed thyrotoxicosis were ANCA-negative before commencement of carbimazole. One (10\%) became ANCApositive within 8 months of therapy.

Conclusions: In our population, ANCA-positivity in association with long-term anti-thyroid medication is common $(26.7 \%)$. One patient who was ANCA-negative prior to anti-thyroid therapy has become ANCA-positive. ANCA should be tested in patients receiving long-term anti-thyroid medications, and in patients with adverse reactions. As PTU is more commonly associated with vasculitis and positive ANCA, carbimazole may be the preferred medication for long-term use. Patients with positive ANCA should be followed, and considered for definitive anti-thyroid therapy, to allow cessation of medication. ANCA-positivity may resolve after cessation of anti-thyroid medication.
\end{abstract}

European Journal of Endocrinology 142 587-590

\section{Introduction}

Anti-neutrophil cytoplasmic antibody (ANCA) is found in two forms - cytoplasmic (cANCA) and perinuclear (pANCA). cANCA is associated with antibodies directed against proteinase-3 (PR3), and is the form commonly seen in Wegener's granulomatosis. pANCA can be directed against a variety of antigens, and is commonly associated with antibodies directed against myeloperoxidase (MPO).

There are 32 reported cases of ANCA-positive vasculitis in the English literature in association with anti-thyroid medication (1-10). ANCA-positive vasculitis in association with anti-thyroid drugs was first reported in 1992 (5). The presenting symptoms are variable and may include renal involvement (67\%), arthralgia $(48 \%)$, fever $(37 \%)$, skin involvement $(30 \%)$, respiratory tract involvement $(27 \%)$, myalgia $(22 \%)$, scleritis $(15 \%)$ and other manifestations $(18 \%)$. Underlying thyroid disease is most commonly Graves' disease, but ANCA-positive vasculitis has also been seen in association with toxic multi-nodular goitre (1). Most cases have occurred in those patients receiving long term (>18 months) therapy with anti-thyroid medications, or with a recent commencement. Approximately $90 \%$ of cases have occurred in association with propylthiouracil (PTU). Seventy-five per cent of cases have occurred in female patients, and approximately half of reported cases have occurred in Japanese patients. There are few data for the frequency of positive ANCA in patients taking anti-thyroid medications. 
Table 1 Demographic characteristics.

\begin{tabular}{lll}
\hline & \multicolumn{2}{c}{ Anti-thyroid therapy } \\
\cline { 2 - 3 } & No previous & \multicolumn{1}{c}{ Long-term } \\
\hline Number & 10 & 30 \\
Sex (M/F) & $2 / 8$ & $2 / 28$ \\
Ethnicity (Caucasian/Asian) & $3 / 7$ & $27 / 3$ \\
Age (years) & $46 \pm 15$ & $54 \pm 31(P=0.055)$ \\
Graves' disease & 7 & 19 \\
CBZ (median, years) & 0.5 & 1.5 \\
CBZ (mean, years) & $0.54 \pm 0.18$ & $1.65 \pm 3.7$ \\
PTU (median, years) & 0 & $1.5(P=0.002)$ \\
PTU (mean, years) & 0 & $3.1 \pm 8.9$ \\
Total (median, years) & 0.5 & $3.0(P<0.001)$ \\
Total (mean, years) & $0.54 \pm 0.18$ & $4.7 \pm 8.1$ \\
\hline
\end{tabular}

CBZ, carbimazole, Total, total duration of any anti-thyroid medication.

\section{Subjects, materials and methods}

Because most of the cases of ANCA-positive vasculitis have occurred in the setting of long-term use, or recent commencement of therapy, two groups of patients were assessed. ANCA was tested in patients taking long-term (>18 months) anti-thyroid medication who attended clinic between 28 April 1998 and 30 September 1998. Thirty patients receiving long-term therapy with antithyroid medications were tested. Patients with a total duration of therapy $<18$ months were not included in this group.

A separate group of ten consecutive patients presenting to clinic between 28 April 1998 and 30 September 1998 with newly diagnosed thyrotoxicosis were tested for ANCA prior to commencing anti-thyroid medication, and after $\geq 6$ months of therapy with carbimazole.

For patients in both groups, data were obtained about sex, ethnicity, age, anti-thyroid medication, duration of therapy and underlying thyroid disease. Manifestations of possible adverse effects of anti-thyroid medications were noted. ANCA was repeated 4-6 months after any change in therapy.

ANCA was performed by direct immunofluorescence with human neutrophils. The pattern of staining was classified as cANCA or pANCA, and cANCA-positive specimens were further tested for antibody to PR 3 by enzyme immunoassay (PR3-ANCA, Euro-Diagnostica AB, Ideon SE-205 12, Malmo, Sweden). pANCApositive specimens were tested for antibody specificity against myeloperoxidase (MPO) by enzyme immunoassay (MPO-ANCA, Euro-Diagnostica AB, Ideon SE-205 12, Malmo, Sweden). Means are stated \pm 2 s.D. Duration of therapy was not normally distributed, and was tested as a non-parametric variable. Statistical analysis was performed with SPSS.

\section{Results}

Patient characteristics are shown in Table 1.

Eight of ten $(80 \%)$ newly diagnosed patients were female, and 28 of 30 (93.3\%) patients on long-term therapy tested were female. Seventy per cent of the newly diagnosed patients were of Asian ethnic origin, and $10 \%$ of the long-term therapy group. Mean age did not differ significantly between the groups.

None of the ten patients with newly diagnosed thyrotoxicosis were ANCA-positive $(0 \%)$ prior to therapy. All were commenced on carbimazole therapy. One

Table 2 Characteristics of ANCA-positive patients.

\begin{tabular}{|c|c|c|c|c|c|c|c|c|}
\hline & Age, Sex & $\begin{array}{c}\text { Therapy } \\
\text { duration } \\
\text { (years) }\end{array}$ & $\begin{array}{l}\text { Thyroid } \\
\text { disease }\end{array}$ & ANCA titre and type & $\begin{array}{l}\text { MPO } \\
(<10)\end{array}$ & $\begin{array}{l}\text { PR3 } \\
(<10)\end{array}$ & $\begin{array}{c}\text { Symptoms and signs } \\
\text { at testing }\end{array}$ & Medication and follow-up ANCA \\
\hline 1 & $48, F$ & $\begin{array}{l}5 \text { PTU, } \\
1 \mathrm{CBZ}\end{array}$ & Graves' & 160 pANCA & 18 & $<1$ & Myalgia arthralgia & Ceased, ANCA + \\
\hline 2 & $61, F$ & $\begin{array}{l}4 \text { PTU, } \\
3 \text { CBZ }\end{array}$ & Graves' & 80 pANCA & $<10$ & $<10$ & Arthralgia haematuria & Ceased, ANCA - \\
\hline 3 & $27, F$ & $\begin{array}{l}4 \text { PTU, } \\
3 \text { CBZ }\end{array}$ & Graves' & $>160$ pANCA cANCA & 45 & 30 & Arthralgia malaise & Ceased, ANCA + \\
\hline 4 & $55, F$ & 8 PTU & MNG & $>160$ pANCA & 14 & $<1$ & Worsened CMP & Ceased, ANCA - \\
\hline 5 & $69, \mathrm{~F}$ & 13 PTU & MNG & $>160$ pANCA & 13 & $<10$ & Past rash, cough & Ceased, ANCA + \\
\hline 6 & $48, F$ & 15 PTU & Graves' & 160 pANCA & 40 & $<10$ & None & Ceased, ANCA - \\
\hline 7 & $35, F$ & 13 PTU & Graves' & $>640$ pANCA cANCA & 16 & 11 & None & Ceased PTU for CBZ, ANCA + \\
\hline 8 & $76, F$ & $\begin{array}{r}1.5 \\
\mathrm{CBZ}\end{array}$ & MNG & $80 \mathrm{pANCA}$ & 11 & $<10$ & None & Ceased, ANCA - \\
\hline $9^{*}$ & $34, F$ & $\begin{array}{l}0.75 \\
\mathrm{CBZ}\end{array}$ & Graves' & 40 cANCA (atypical) & 37 & $<10$ & None & Continues therapy \\
\hline
\end{tabular}

PTU, propylthiouracil; MNG, multinodular goitre; CBZ, carbimazole; CMP, dilated cardiomyopathy.

* Patient 9 was ANCA negative before CBZ therapy, and became ANCA positive by 8 months of therapy. 
patient (10\%) became ANCA-positive after 8 months of therapy (patient 9 in Table 2). Nine patients remain ANCA-negative after $\geq 6$ months of therapy.

Positive ANCA was found in 8 of 30 patients receiving long-term anti-thyroid medication (Table 2). Mean age of ANCA-positive patients was not significantly different from ANCA-negative patients $(56.6 \pm 31.4$ versus $55.1 \pm 30.9$ years). All ANCA-positive patients were female, so sex distribution was not tested for significance. Twenty of the 22 (90.9\%) ANCA-negative patients were Caucasian, and the remainder were Asian $(9.1 \%)$. Of the eight ANCA-positive patients, seven $(87.5 \%)$ were Caucasian, and one was Asian. Five ANCA-positive patients had underlying Graves' disease $(62.5 \%)$, and three $(37.5 \%)$ had toxic multi-nodular goitre as the underlying cause of thyrotoxicosis, compared with $63 \%$ and $37 \%$ for the ANCA-negative patients.

pANCA was present in seven of eight ANCA-positive patients, and cANCA-positive in two of eight. One patient was positive for both cANCA and pANCA. Of the pANCA-positive patients, six were MPO-positive. Of the two cANCA-positive patients, one was strongly PR3-positive, and one equivocally positive.

Seven of eight patients with positive ANCA were receiving PTU at the time of testing (for a mean duration of $7.9 \pm 10.2$ years). Three of these seven also had past exposure to carbimazole. One ANCA-positive patient had received only carbimazole. Of the ANCAnegative patients, $59 \%$ had received carbimazole alone, 23\% PTU alone, and 18\% both medications. Patients with positive ANCA received anti-thyroid medications for a mean duration of $8.8 \pm 9.0$ years (median 6.5 years), compared with a mean duration of $2.8 \pm 4.0$ years (median 2.5) in the ANCAnegative patients (Mann-Whitney, $P=0.002$ ). The mean duration of PTU therapy was $7.9 \pm 10.2$ years (median 6.0) in ANCA-positive patients, compared with $0.8 \pm 2.2$ years (median 0.5) in the ANCA negative patients $(P<0.0001)$. The mean duration of carbimazole therapy was $0.9 \pm 2.6$ years (median 0.5 ) in the ANCA-positive patients, compared with $2.0 \pm 4.0$ years (median 2.0) in the ANCA-negative patients $(P=0.11)$.

Four ANCA-positive patients had symptoms which may have been due to anti-thyroid medication. Three patients (Table 2, patients 1, 2 and 3) had arthralgia and myalgia, with symptoms commencing $4.5,3.75$ and 2 years respectively after commencing PTU. At the time of testing, the symptoms had been present for 6, 9 and 22 months respectively. Symptoms resolved with cessation of anti-thyroid medication in all of these patients. Patient 4 had a past history of purpuric rash which settled despite continued therapy.

Five of the eight ANCA-positive patients have become ANCA-negative by 6 months after cessation of medication (patients 2, 3, 5, 6 and 8).

One patient with a negative ANCA during the study period had previously tested positive. This was a pANCA at a titre of 1:160, MPO-positive at 24 ( $<10$ units), and PR3 negative ( $<10$ units). She was a 44-year-old woman with Graves' disease who received PTU for 18 months. As a result of the positive ANCA, therapy was switched to carbimazole. Four months after this change in therapy, ANCA was negative.

\section{Discussion}

ANCA-positive vasculitis in association with antithyroid drugs was first reported in 1992 (5). To our knowledge, 32 cases have been reported in the English literature (1-10). The majority of cases (88\%) have occurred in association with PTU. However, the vasculitis has also been seen with carbimazole (4). ANCA-positive vasculitis has been seen in association with other medication, including hydralazine $(11,12)$, sulphasalazine $(13,14)$ and minocycline $(15)$.

The pathogenesis is not clearly understood. PTU has been shown to accumulate within neutrophils (16) and bind myeloperoxidase (17). The binding alters the configuration of myeloperoxidase (17), and may promote formation of autoantibodies in susceptible people. In cats, treatment with PTU has been shown to induce ANCA, and a diet high in taurine appears to prevent this (18). There are no data with regard to whether carbimazole can alter the configuration of myeloperoxidase. A highly significant association between duration of therapy with PTU and positive ANCA was found $(P<0.0001)$, but there was no significant association with duration of carbimazole therapy.

ANCA-positive vasculitis may be more common in patients of Asian ethnic origin, with half of the cases reported English literature from Japan, and further cases in the Japanese literature. The high proportion of the newly diagnosed patients from an Asian ethnic background may have provided a bias towards development of positive ANCA in this study.

Among our population of patients on long-term anti-thyroid medication, positive ANCA is common (26.7\%). Seven of the eight ANCA-positive long-term medication patients have ceased anti-thyroid medications. The eighth ceased medication but had a relapse of Graves' disease during pregnancy, and was treated with carbimazole. The pregnancy was otherwise uneventful and she was delivered of a normal infant at 37 weeks gestation. Five of eight ANCA-positive patients have become ANCA-negative since ceasing medication.

Negative results before commencement of therapy in ten patients make it less likely that ANCA is caused by underlying thyroid disease, or by cross-reactivity with other thyroid antibodies.

The fulminant form of ANCA-positive vasculitis in association with anti-thyroid medication is rare. However, positive ANCA may be related to or be a marker of adverse reactions to anti-thyroid medications. 
Patients with positive ANCA may be a subgroup more likely to develop ANCA-positive vasculitis.

The authors think that ANCA should be tested in those patients receiving long-term (more than 18 months) anti-thyroid medication. Patients who develop symptoms or signs on anti-thyroid medication should also have ANCA measured. It may be appropriate to have a lower threshold for testing patients of Asian ethnic origin; however, in our Caucasian patients, $\mathrm{ANCA}(+)$ is common. A positive ANCA may influence clinical management by leading to cessation of medication, or administration of definitive therapy. Given an otherwise similar side-effect profile, it may be reasonable to select carbimazole as initial therapy.

\section{Acknowledgements}

The authors wish to thank the Immunology laboratory at Pacific and Laboratory Medicine Services (PaLMS) for performing ANCA testing.

\section{References}

1 Gunton JE, Stiel J, Caterson RJ \& McElduff A. Anti-thyroid drugs and antineutrophil cytoplasmic antibody positive vasculitis. A case report and review of the literature. Journal of Clinical Endocrinology and Metabolism 199984 13-16.

2 Dolman KM, Gans ROB, Vervaat TJ, Zevenbergen G, Maingay D, Nikkels RE et al. Vasculitis and antineutrophil cytoplasmic autoantibodies associated with propylthiouracil therapy. Lancet $1993342651-652$.

3 Vogt BA, Kim Y, Jennette JC, Falk RJ, Burke BA \& Sinaiko A. Antineutrophil cytoplasmic autoantibody-positive crescentic glomerulonephritis as a complication of treatment with propylthiouracil in children. Journal of Pediatrics 1994 124 986-988.

4 D'Cruz D, Chesser AMS, Lightowler C, Comer M, Hurst MJ, Baker LR et al. Antineutrophil cytoplasmic antibody-positive crescentic glomerulonephritis associated with anti-thyroid drug treatment. British Journal of Rheumatology 199534 10901091.

5 Stankus SJ \& Johnson NT. Propylthiouracil-induced hypersensitivity vasculitis presenting as respiratory failure. Chest 1992102 1595-1596.

6 Ohtsuka M, Yamashita Y, Doi M \& Hasegawa S. Propylthiouracil- induced alveolar haemorrhage associated with antineutrophil cytoplasmic antibody. European Respiratory Journal 199710 1405-1407.

7 Miller RM, Savige J, Nassis L \& Cominos BI. Antineutrophil cytoplasmic antibody (ANCA)-positive cutaneous leucocytoclastic vasculitis associated with antithyroid therapy in Graves' disease. Australasian Journal of Dermatology 199839 96-99.

8 Harper L, Cockwell P \& Savage COS. Case of propylthiouracilinduced ANCA associated small vessel vasculitis. Nephrology Dialysis Transplantation 199813 455-458.

9 Almoroth G, Enestrom S, Hed J. Samuelsson I \& Sjostrom P. Autoantibodies to leucocyte antigens in hydralazine-induced nephritis. Journal of Internal Medicine 1992231 37-42.

10 Pillinger M \& Stand R. Wegener's granulomatosis in a patient receiving propylthiouracil for Graves' disease. Seminars in Arthritis and Rheumatism 199828 124-129.

11 Fujieda M, Nagata M, Akioka Y, Hattori M, Kawaguchi H \& Ito K. Antineutrophil cytoplasmic antibody-positive crescentic glomerulonephritis associated with propylthiouracil therapy. Acta Paediatrica Japonica 199840 286-289.

12 Nassberger L, Sjoholm AG, Jonsson H, Sturfelt G \& Akesson A. Autoantibodies against neutrophil cytoplasm components in systemic lupus erythematosus and in hydralazine-induced lupus. Clinical Experimental Immunology 199081 380-383.

13 Salerno SM, Ormseth EJ, Roth BJ, Meyer CA, Christensen ED \& Dillard TA. Sulfasalazine pulmonary toxicity in ulcerative colitis mimicking clinical features of Wegener's. Chest 1996110 556-559.

14 Hill ME, Gordon C, Situnayake RD \& Heath DA. Sulfasalazine induced seizures and dysphasia. Journal of Rheumatology 199421 748-749.

15 Elkayam O, Yaron M \& Caspi D. Minocycline induced arthritis associated with fever, livedo reticularis, and pANCA. Annals of Rheumatic Diseases 199655 769-771.

16 Lam DCC \& Lindsay RH. Accumulation of 2-[ $\left.{ }^{14} \mathrm{C}\right]$-propylthiouracil in human polymorphonuclear leukocytes. Biochemical Pharmacology 197928 2289-2296.

17 Lee H, Hirouchi M, Hosokawa M, Sayo H, Kohno M \& Kariya K. Inactivation of peroxidase of rate bone marrow by repeated administration of propylthiouracil is accompanied by a change in the heme structure. Biochemical Pharmacology 198837 2151-2153.

18 Waldhauser L \& Uetrecht J. Antibodies to myeloperoxidase in propylthiouracil-induced autoimmune disease in the cat. Toxicology 1996114 155-162.

Received 21 July 1999

Accepted 7 February 2000 